FORMATION Formation emploi

Revue française de sciences sociales

99 | juillet-septembre 2007

Les usages sociaux de la compétence

Lucie Tanguy, Les instituts du travail. La formation syndicale à l'université de 1955 à nos jours

(2006), Presses universitaires de Rennes, Rennes, 256 p.

\title{
Christian Marquette
}

\section{OpenEdition}

Journals

Édition électronique

URL : http://journals.openedition.org/formationemploi/1527

DOI : 10.4000/formationemploi. 1527

ISSN : 2107-0946

Éditeur

La Documentation française

Édition imprimée

Date de publication : 1 juillet 2007

Pagination : 143-149

ISSN : 0759-6340

Référence électronique

Christian Marquette, "Lucie Tanguy, Les instituts du travail. La formation syndicale à l'université de 1955 à nos jours », Formation emploi [En ligne], 99 | juillet-septembre 2007, mis en ligne le 19 février 2009, consulté le 30 octobre 2020. URL : http://journals.openedition.org/formationemploi/1527 ; DOI : https://doi.org/10.4000/formationemploi.1527

(c) Tous droits réservés 


\section{sercture \\ Note}

\section{Les instituts du travail. La formation syndicale à l'université de 1955 à nos jours}

Par Christian Marquette*

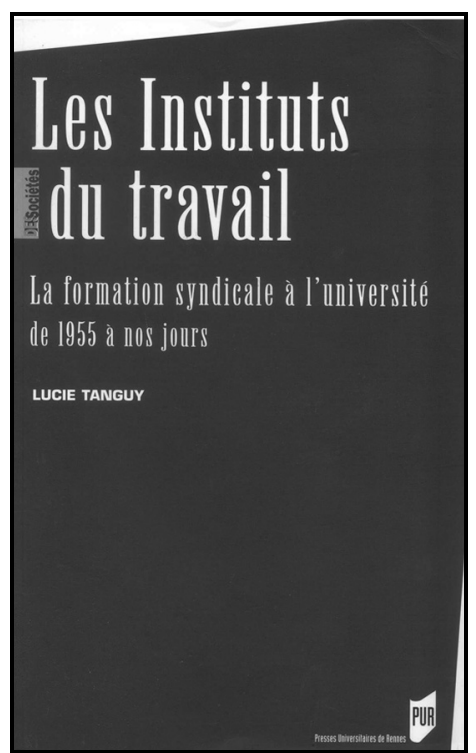

L'ouvrage que nous propose Lucie Tanguy s'inscrit dans une recherche collective consacrée à l'histoire de la formation permanente et à la diffusion de certains de ses modèles dans la société française d'après 1970. Il porte sur une réalisation particulière que sont les Instituts du travail.

Créés à partir de 1955, les Instituts du travail sont destinés à offrir une formation supérieure aux cadres et délégués syndicaux des confédérations ouvrières :

la Confédération française démocratique du travail (CFDT), la Confédération générale du travail (CGT) et la Confédération générale du travail - Force ouvrière (CGT-FO). Selon les vœux de leur principal fondateur, Marcel David, ils visent la préparation des syndicalistes à l'exercice du droit, à la gestion d'organismes sociaux, et plus généralement à la compréhension des changements socio-économiques en cours.

Il existe actuellement onze Instituts du travail, répartis sur tout le territoire. Deux de ces Instituts, à
Paris et à Strasbourg, les premiers à avoir été créés, ont un caractère national et les neuf autres un caractère régional. Dans leur ensemble, ils accueillent environ 4000 stagiaires chaque année. Les 12 et 13 octobre 2006, un colloque organisé à Strasbourg sur le thème «Le salarié, acteur de son évolution professionnelle - Mythe ou réalité ?» a été l'occasion de marquer les 50 ans du premier Institut. Il a permis aux participants de souligner la fécondité du

Christian Marquette est chargé d'études au Céreq. II a dirigé pendant de nombreuses années la Fédération Chimie de la CFDT avant de conduire, depuis 1990, des travaux d'ordre sociologique se rapportant au travail et à la formation professionnelle, et en particulier à l'apprentissage et la transmission des savoirs d'action. Après des travaux portant sur la diffusion des procédures d'assurance qualité dans le secteur industriel, il a récemment mené des investigations à ce sujet dans le milieu hospitalier. II a notamment publié "Une rationalisation sans norme organisationnelle: la certification ISO 9000 », avec M. Campinos-Dubernet, dans Sciences de la société, $n^{\circ} 46,1999$ et « L'histoire longue de la mise en œuvre d'un accord sur les compétences: quelques pistes d'analyse », avec P. Kalck et S. Montchatre, in Gestion des compétences - Acteurs et pratiques, coordonné par D. Brochier, Économica, 2002. 
partenariat engagé entre l'Université et les organisations syndicales, de mesurer le chemin parcouru de 1955 à nos jours et de s'interroger sur un nouvel élan à donner à ces institutions pour les rendre à même de jouer pleinement leur rôle dans le nouveau contexte que nous connaissons de nos jours.

La rétrospective historique et les analyses rigoureuses que nous livre Lucie Tanguy au fil de l'ouvrage retiendront l'attention de tous ceux qui s'intéressent à la formation tout au long de la vie. Si, de nos jours, ces institutions de formation souffrent d'une moindre visibilité sociale, leur expérience passée et présente peut enrichir bien des réflexions contemporaines se rapportant à la validation des acquis de l'expérience, aux relations entre universités et mondes du travail, à la confrontation entre savoirs retirés de l'action et savoirs académiques...

La création des Instituts du travail, leurs fonctionnements et leurs réalisations ont constitué des contributions singulières tant au regard des pratiques de formation permanente ou d'éducation populaire développées à la même époque dans d'autres lieux, qu'au regard aussi du fonctionnement traditionnel des universités. Dans les années 50, des certificats portant sur des matières sociales et économiques ouverts aux militants ouvriers avaient déjà été introduits au sein de l'Université mais ces cours ne dérogeaient d'aucune façon à la forme universitaire. À l'initiative d'universitaires, en rupture avec les pédagogies scolaires, il s'est agi d'élaborer un modèle original visant la constitution d'un savoir non plus individualisé mais de groupe qui, dans l'acquisition des connaissances, accorde la primauté à l'expérience retirée de l'action militante. Dès lors, dans le cadre universitaire, il fallait faire vivre une institution en collaboration avec des syndicalistes qui revendiquent leur autonomie de pensée ainsi que le contrôle de la formation délivrée à leurs propres militants, les contenus des formations étant négociés entre syndicats et universitaires puis dispensés de manière croisée par le recours à des équipes enseignantes qui réunissent des intervenants des deux partis.

Au travers des différents chapitres de son ouvrage, Lucie Tanguy retrace l'histoire de cette institution universitaire, "hétéronome » de par son statut et ses règles de fonctionnement. Elle examine les spécificités de la «pédagogie hybride» mise en œuvre pour délivrer un enseignement supérieur qui, confrontant des savoirs universitaires à des expériences militantes, participe de l'émancipation des militants syndicaux tout autant qu'elle vise à renforcer la transformation des rapports sociaux et de la société. De même, elle consacre utilement l'une des sections de son ouvrage aux auteurs des actions collectives et individuelles qui ont rendu possible la réalisation des Instituts, et principalement aux universitaires qui en ont eu l'initiative. Elle y souligne le rôle qu'ont pu jouer, dans la création des Instituts, et plus largement dans la promotion de la « formation permanente», des intellectuels engagés appartenant « aux milieux chrétiens de gauche ou de catholiques sociaux ».

En elles-mêmes, la rétrospective historique, les informations et les analyses proposées dans l'ouvrage constituent déjà des apports appréciables pour une meilleure connaissance de la formation syndicale délivrée dans le cadre universitaire. Mais ce qui rend particulièrement précieux ce travail de Lucie Tanguy c'est que l'histoire des Instituts du travail y est ici étudiée comme un chapitre de l'histoire sociale de la France de ce dernier demi-siècle. En effet, ainsi que le remarque l'auteure dans la présentation de son travail, "l'histoire des Instituts du travail n'est pas seulement celle d'une institution de formation des cadres du syndicalisme. [...] (Elle) peut être considérée comme une expérience de démocratisation $d u$ savoir qui tentait d'établir des liens entre pensée et action politique, d'une manière très différente de celle des intellectuels contemporains qui étaient convaincus que l'accès à la connaissance pouvait bouleverser le monde ».

Dans cette note de lecture, nous voudrions souligner le vif intérêt que présente l'approche ainsi adoptée pour étudier les Instituts du travail. Mais nous voudrions le réaliser d'une manière particulière, celle du reste valorisée par ces institutions de formation, puisqu'il s'agira de croiser les résultats de la recherche de Lucie Tanguy avec quelques-uns des enseignements retirés de l'expérience d'acteurusager des Instituts du travail, qui a été la notre au cours des décennies 70 et 80 . En effet, nous avons été stagiaire dans les Instituts de Strasbourg et de Paris puis responsable de la Fédération CFDT des 
industries chimiques et co-organisateur de sessions de formation-recherche avec les universitaires de ces deux mêmes instituts.

Comme ce fut généralement le cas de nombreux militants syndicaux de cette époque, nous n'avons bénéficié d'autres enseignements supérieurs que ceux délivrés par les Instituts du travail. Toutefois, il nous faut souligner qu'en aucun cas les stages suivis dans le cadre universitaire n'auront été vécus par nous comme des actions de formation permanente ayant des visées à caractère individuel en matière d'acquisition de connaissance comme de promotion sociale.

Mais avant de définir la fonction que ces sessions de formation-recherche remplissaient au sein du syndicalisme pratiqué par la Fédération Chimie CFDT et de faire ainsi écho aux propositions de Lucie Tanguy, nous souhaitons aventurer quelques pistes de réflexion concernant le rôle décisif qui, d'après l'auteure, aura été rempli par des intellectuels d'appartenance «chrétiens de gauche». Il ne s'agit pas de discuter de ce fait assez bien établi mais plutôt de s'interroger sur la lecture qu'il convient d'en faire pour non seulement mieux comprendre le contexte qui a présidé à la création de ces institutions universitaires mais aussi tenter de mettre au jour les enjeux qui les concernent aujourd'hui.

Tout d'abord, en nous référant à l'expérience que nous avons directement vécue au sein du syndicalisme CFDT, si nous rapprochons les actions innovantes en matière de formation économique et sociale initiées par Marcel David de celles, non moins singulières, qu'aura impulsées Alain Wisner dans le domaine des conditions de travail et de l'ergonomie (Teiger et alii, 2006), nous ne pouvons manquer d'être frappés par les similitudes. Dans les deux cas, ces innovations, engagées à l'initiative d'intellectuels issus de milieux chrétiens de gauche, caressent une même audacieuse ambition : instituer des lieux d'échange et d'élaboration d'un type de savoir résultant de la recherche scientifique des intellectuels et de l'action collective des militants syndicaux. Autrement dit, permettre aux militants de réfléchir leurs pratiques et de se doter d'instruments susceptibles d'accroître l'efficacité de leurs actions et, dans le même temps, permettre aux universitaires de confronter leurs concepts théoriques aux pratiques syndicales. Aussi, bien que l'on puisse considérer l'action des fondateurs des Instituts du travail comme une composante d'un « vaste mouvement social pour la formation ", ainsi que le fait Lucie Tanguy, c'est bien plus les caractéristiques que nous venons d'évoquer plus haut qui, à notre sens, spécifient les innovations introduites dans les années 50 au sein de l'Université. Mais, en définitive, c'est à une telle lecture que nous semble bien inviter l'auteure.

Quant à l'appartenance de ces pionniers «aux milieux chrétiens de gauche ou de catholiques sociaux » qui, souligné par Lucie Tanguy, paraît faire problème du côté de certains des universitaires des Instituts du travail ${ }^{1}$, nous aurions tendance à ne pas lui attribuer une trop grande importance quant à ses incidences sur les finalités de ces institutions. D'ailleurs, à ce sujet aussi, les analyses qui nous sont proposées paraissent fort peu ambiguës. Si l'auteure observe que les fondateurs d'une formation syndicale à l'université appartiennent effectivement à l'élite intellectuelle qui considère la formation comme l'un des principaux leviers de la modernisation du pays, elle ne distingue pas moins très nettement la perspective engagée qu'ils choisissent résolument, de leur côté, de celle envisagée par la majorité de cette élite. Pour celle-ci, selon Lucie Tanguy, la formation est "utilisée comme un moyen de faire adhérer tous les membres d'une organisation ou de la société aux objectifs affichés de celle-ci: la coopération, la participation, la négociation au lieu et place de la confrontation sociale et politique qui caractérisait les décennies d'après-guerre» tandis que «les universitaires des instituts, eux, inscrivent leur action dans un projet de changement plus radical de la société dont les syndicats sont acteurs ». Et c'est à l'évidence en cela, conjugué à un ensemble d'éléments factuels et contextuels, que réside une sorte de secret de réussite d'une entreprise que beaucoup considéraient vouée à un échec quasi certain.

Pour notre part, nous ajouterons ici un point d'histoire. L'ample travail conduit par Lucie Tanguy

\footnotetext{
${ }^{1}$ Si l'on en juge en effet aux contenus ou à la tonalité de quelques interventions réalisées par d'actuels ou d'anciens responsables d'Instituts du travail à l'occasion du colloque du cinquantième
} anniversaire de cette institution qui s'est tenu à Strasbourg. 
considère uniquement la période qui va de 1955 à nos jours, c'est-à-dire celle au cours de laquelle ont vu le jour et se sont développés les actuels Instituts $\mathrm{du}$ travail. Mais il n'est pas indifférent de savoir qu'un projet similaire avait été nourri aux lendemains même de la première guerre mondiale. Dans un essai, publié en 1927, intitulé «Les Instituts du travail dans nos universités », sur une centaine de pages qui regroupent une série de huit articles de revue publiés sept années auparavant, l'intellectuel Charles Andler ${ }^{2}$, professeur au Collège de France, développe un projet offrant d'étonnantes ressemblances avec l'innovation qui verra effectivement le jour plus de trente ans après. De même que dans le projet abouti de Marcel David, il est question d'un haut enseignement ouvrier pour à la fois contribuer à l'émancipation des ouvriers et leur permettre, selon Andler, d'être mieux à même de contribuer à la modernisation en faisant face aux «modifications profondes que vient de subir l'outillage» ainsi qu'à celles induites par "la réglementation taylorienne des usines ». De même, il y est question d'un «Institut du travail qui n'enseigne pas autre chose que ce qui fait l'objet de l'enseignement traditionnel de nos Universités et de nos grandes écoles. Mais il l'enseigne d'une façon nouvelle. Il se propose une autre fin qu'il ne doit pas perdre de vue et qui, à elle seule, est novatrice ».

Nous ignorons cependant s'il existe des filiations entre ce projet et celui porté par un autre groupe d'intellectuels, un tiers de siècle plus tard. Constatons seulement que Marcel David n'en fait guère état dans l'analyse de son expérience de coopération entre universitaires et militants syndicalistes qu'il a publiée en 1982 sous le titre «Témoins de l'impossible - militants du monde ouvrier à l'université ».

Comme Lucie Tanguy en fait un rappel, dans son ouvrage, Marcel David a exposé les attitudes de méfiance, puis de réserve et enfin d'adhésion hésitante des confédérations ouvrières au projet de formation universitaire qu'il élaborait à leur

\footnotetext{
2 Charles Andler (1866-1933), germaniste tout comme son ami Lucien Herr (1864-1926), le très prestigieux et influent bibliothécaire de l'École normale, membres de la génération qui a refondé l’Université en France, ont travaillé dans les domaines sociaux et intellectuels aux côtés de grandes figures universitaires ou politiques tels que Durkheim, Mauss, Simiand, Jaurès ou Blum.
}

intention. $\mathrm{La} \mathrm{CFTC}^{3}$ y a été plutôt vite acquise en raison de l'importance qu'elle accorde de longue date à la formation et au développement personnel en tant que composante de l'action collective. La CGT et la CGT-FO manifesteront, au début, un peu plus de prévention envers une telle initiative extérieure. En dépit des réserves affichées par les syndicats, ce projet rencontrait cependant certaines de leurs attentes en venant relayer, comme pour la CGT par exemple, la tentative d' « école de cadres » qu'elle avait envisagée. Par la suite, les formations codispensées par les Instituts, résultant de processus de négociation entre syndicalistes et universitaires, chaque organisation syndicale, et même chacune de leurs propres structures, imprimera une coloration venant refléter les orientations et les stratégies d'action qui lui sont propres. Cette évolution n'étant d'ailleurs pas celle envisagée à l'origine par les fondateurs des Instituts qui, en quête d'une unification du mouvement syndical, souhaitaient que leur initiative contribue en contrecoup à la réduction des clivages syndicaux.

En sachant donc que chaque situation étudiée constitue un cas d'espèce non généralisable, nous voudrions maintenant confronter des expériences vécues au sein de la fédération chimie de la CFDT aux analyses de Lucie Tanguy. Nous souhaiterions en fait prolonger certains aspects de son travail en montrant comment les usages qu'une organisation professionnelle de branche a pu faire des dispositifs de formation des Instituts du travail, auront été très proches dans leurs principes de ceux qui fondaient le projet initié par Marcel David.

C'est dans les années 50, au moment même ou Marcel David concevait les Instituts du travail, que la fédération chimie de la CFDT définissait sa propre politique de formation syndicale. Pour les responsables de cette organisation, comme le relève Guy Brucy (1999), la formation n'avait de sens que si on la comprenait comme un des éléments contribuant à la construction de la «force contractuelle» que devait constituer le syndicalisme. Envisagée de la sorte, la formation des militants entend dépasser le

\footnotetext{
${ }^{3}$ La CFTC, Confédération française des travailleurs chrétiens, deviendra la CFDT en 1964 à la suite d'un processus majoritaire de déconfessionnalisation. Cependant, une petite minorité conservera le sigle CFTC.
} 
seul apprentissage de techniques syndicales et plus largement de techniques sociales et économiques. Elle vise bien à créer les conditions de l'émancipation des travailleurs par une «éducation méthodique » qui favorise chez les syndicalistes, et plus largement pour l'ensemble de la classe ouvrière, «le développement d'une large culture».

Dans les décennies 70-80, période syndicale que nous avons vécue, pour tenter de répondre aux mutations économiques et sociales suscitées par la crise, les orientations traditionnelles de la fédération en matière de formation/recherche ont connu une sorte de second souffle. Il s'est alors agi de développer des réflexions approfondies autour de l'organisation du travail, de l'emploi et des questions industrielles, tant techniques que sociales et économiques. L'objectif n'était pas celui d'une meilleure compréhension des évolutions en cours, en vue de la contestation des choix patronaux ou gouvernementaux. De manière plus ambitieuse, cette recherche visait l'élaboration de propositions syndicales cohérentes à opposer aux projets des employeurs afin de ne pas être tenus à l'écart, de peser sur l'orientation des transformations, de limiter l'arbitraire patronal et de favoriser l'implication des salariés dans des domaines qui les concernent directement. Bref, ainsi que le souligne Guy Brucy (1997), il s'agissait de «proposer pour ne pas subir. Il importait d'élaborer des solutions alternatives porteuses des valeurs ouvrières comme la solidarité, l'autonomie, la capacité à contrôler le processus de travail ». Pour une bonne part, précise l'auteur, un tel travail sur la connaissance était «accompli en collaboration avec des institutions universitaires : université de Grenoble, Institut du travail de Strasbourg et de Sceaux ».

Cela fait, nous semble-t-il, assez bien écho aux finalités de cette «pédagogie hybride» des Instituts que nous expose Lucie Tanguy dans un chapitre de son travail. À savoir : une pédagogie qui doit «permettre au militant d'acquérir une intelligence directe des problèmes [...]. La capacité personnelle à comprendre une situation, à l'interpréter, à la représenter et à faire adhérer les autres à une action dans et sur cette situation résulte d'une alchimie où l'expérience est prépondérante. La compétence technique, dans ce type de formation, peut être résumée comme une capacité d'exprimer ceux que le militant représente».

Mais, selon l'auteure, cette relation d'enchantement qui sous-tendait la formation «émancipatrice» réalisée dans les Instituts du travail aurait peu à peu laissée place à une relation instrumentale entre universitaires et syndicalistes : «le public et acteur de ces instituts, les syndicats, qui était supposé confronter son savoir d'expérience au savoir savant des universitaires, n'est plus porteur d'un projet pédagogique fort. Son rapport aux instituts est plus pragmatique, intéressé et détaché à la fois. En s'institutionnalisant, les syndicats ont abandonné les ambitions théoriques qu'ils revendiquaient antérieurement: donner une interprétation synthétique du monde social sur lequel et dans lequel ils agissent». De ce fait, "d'une formation collective qui, à l'origine, ne connaissait les militants qu'à partir de leurs organisations, elle s'adresse aujourd'hui à des militants qui ne se pensent plus seulement comme des défenseurs d'une cause collective mais comme des individus susceptibles de convertir leurs compétences d'organisation, de mobilisation, de communication sur un marché de cadres de l'économie sociale».

L'ouvrage ne se voulait pas une simple rétrospective historique des Instituts du travail mais bien plus une analyse de ces institutions de formation en tant que chapitre de l'histoire sociale de la France. Le projet de Lucie Tanguy nous semble doublement atteint.

Il l'est tout d'abord en raison des faits rassemblés et des analyses proposées qui nous montrent comment des « convergences équivoques d'intérêts » ont pu, à la faveur du contexte de modernisation de l'aprèsguerre, autoriser et porter une innovation aussi singulière; une innovation qui est alors moins une conquête ouvrière que le résultat des actions d'élites culturelles.

Le projet de l'auteure nous semble aussi atteint par le fait qu'un tel travail, par-delà certains éclairages de notre passé, par-delà aussi des interrogations ayant trait au devenir des Instituts du travail, suscite bien des questions toujours vives touchant à la modernisation et à la réalisation de réformes ainsi qu'à la démocratie et aux relations entre autonomie et intégration des acteurs. 
En citant Michel Hastings, Lucie Tanguy observe que les Instituts du travail se caractérisent par une hétéronomie, attachée à l'histoire du mouvement ouvrier en France, qui s'est bâtie contre le consensus et sur un certain partage du monde. Ce faisant, ce positionnement des organisations ouvrières a aussi fait connaître que le droit à la discorde est le fondement essentiel de la démocratie. Le risque étant grand aujourd'hui de dissoudre l'exception militante dans l'aspiration actuelle au consensus. Un propos que nous faisons pleinement notre à la suite de Jacques Rancière ${ }^{4}$ pour qui, dans un même ordre d'idée, il y a démocratie s'il y a "manifestation d'apparence du peuple [et] conduite du litige sur cette scène [par des] acteurs spécifiques de la politique qui ne sont ni des agents du dispositif étatique ni des parties de la société [...] Ces formes de manifestation ont des effets sur les dispositifs institutionnels du politique et elles se servent de tel ou tel de ces dispositifs [...]. Elles ne sont donc aucunement indifférentes à l'existence d'assemblées élues, de garanties institutionnelles des libertés d'exercice de la parole et de sa manifestation, de dispositifs de contrôle de l'État. Elles y trouvent les conditions de leur exercice et elles les modifient en retour. Mais elles ne s'y identifient pas ».

C'est de la sorte que nous pensons pouvoir interpréter le projet de démocratie sociale que pouvaient caresser les fondateurs des Instituts du travail. C'est aussi, avec le recul, d'une même façon que nous sommes enclins à relire la stratégie de «contrepropositions » que l'organisation syndicale qui a été la notre s'est efforcée de conduire au long des décennies 70-80.

Mais qu'en est-il aujourd'hui ? Comment faut-il comprendre le glissement d'une formation émancipatrice à une formation instrumentale qui affecterait aujourd'hui les Instituts du travail ? Faut-il penser qu'il a comme cause première " l'institutionnalisation, intégration et professionnalisation du syndicalisme » et « l'abandon d'un projet pédagogique fort », ainsi que certains des développements du dernier chapitre de l'ouvrage pourraient le laisser supposer?

${ }^{4} C f$., en particulier, le chapitre « Démocratie ou Consensus » dans l'ouvrage La Mésentente, de Jacques Rancière (2002), pp. 133165.
Ne faut-il pas plutôt penser que ces aspects ne seraient en définitive que les conséquences de la crise des formes de manifestation de notre démocratie, une crise qui prend pour nom « consensus »?

En ce sens, nous aurions tendance à considérer un peu différemment de l'auteure, deux des questions évoquées dans l'ouvrage : celle, tout d'abord, de la fonction d'intégration qu'aurait pu jouer, à leur insu, les Instituts du travail à l'égard du syndicalisme et celle, ensuite, de la promotion de la formation permanente qui serait moins le fruit de conquêtes ouvrières que la résultante d'action d'élites intellectuelles et professionnelles. Aussi distinctes qu'elles soient, à notre sens, ces questions se rapportent, l'une et l'autre, à un seul et même problème qui est celui de la démocratie sociale et de la représentation que l'on peut se faire de son fonctionnement. À la suite d'enseignants de la première génération, dont Lucie Tanguy nous rapporte les interrogations sur le sens de leurs actions passées, est-il bien pertinent de se demander si les actions de formation des Instituts auraient pu «contribuer à intégrer les syndicats en les formant à leurs rôles de représentants des salariés au sein des institutions de gestion sociale et [...] aux fonctions de négociations plus qu'à celles d'affirmations de leurs identités sociales et de leur autonomie»? N'est-ce pas, de la sorte, prendre le risque d'opposer deux niveaux non pas antagoniques mais parfaitement complémentaires de l'intervention syndicale telle qu'elle doit être conduite selon les formes de la démocratie? S'il paraît indéniable que la fondation d'une formation syndicale dans l'université, tout comme la formation permanente, « n'est pas une conquête ouvrière mais la résultante d'action multiples et durables d'élites ouvrant dans diverses sphères de la société », y-a-t'il cependant lieu de s'en étonner? Concernant l'émergence et la diffusion d'innovations touchant à la démocratie sociale, ne faut-il pas former l'hypothèse que cellesci sont moins le produit de conquêtes ouvrières (ou patronales) qu'elles ne résultent de l'émergence de référentiels autour desquels peuvent converger de manière équivoque des intérêts plus ou moins distincts ?

Selon Marc Bloch, qu'aime à citer Lucie Tanguy, "l'ignorance du passé ne se borne pas à nuire à la connaissance du présent, elle compromet, dans le 
présent, l'action même ». Aussi, ce n'est donc pas le moindre des mérites de cette contribution à l'histoire des Instituts du travail que de nous ouvrir la voie à des retours réflexifs sur des problèmes qui concer- nent non seulement l'action de formation des Instituts et des syndicats mais qui ont trait aussi, et autrement plus profondément encore, à la question même de la démocratie sociale.

\section{Référence de l'ouvrage :}

Tanguy L. (2006), Les Instituts du travail - La formation syndicale à l'université de 1955 à nos jours, Presses universitaires de Rennes, Rennes, 256 p.

\section{Bibliographie}

Andler C. (1927), " Les Instituts du travail dans nos universités ", in L'Humanisme travailliste - essai de pédagogie sociale, Bibliothèque de « la civilisation française », Paris, pp. 11-114.

Brucy G. (1997), Histoire de la fédération de la chimie CFDT de 1938 à nos jours, Syros.

Brucy G. (1999), « Formation ou culture, l'action des cadres et des ouvriers de la chimie CFTC-CFDT (1946-1971)», Sociétés contemporaines, $\mathrm{n}^{\circ} 35$, pp. 71-94.

David M. (1982), Témoins de l'impossible, militants du monde ouvrier à l'université, Les Éditions ouvrières.
Guy D. (coord.) (2006), « La formation syndicale universitaire », Les dossiers des sciences de l'éducation, $\mathrm{n}^{\circ}$ 16/2006, Presses universitaires du Mirail.

Palazzeschi (Y. dir.) (2003), « La formation syndicale », Éducation permanente, $n^{\circ} 154 / 1$.

Rancière J. (2002), La Mésentente, «Politique et Philosophie », Galilée.

Teiger C., Barbaroux L. et alii (2006), « Quand les ergonomes sont sortis du laboratoire... à propos du travail des femmes dans l'industrie électronique (1963-1973). Rétro-réflexion collective sur l'origine d'une dynamique de coopération entre action syndicale et recherche-formation-action », Pistes, vol. 8, $\mathrm{n}^{\circ} 2$, octobre. 


\section{$n^{\circ} 15$ \\ Comprendre l'engagement aujourd'hui}

Les engagements ont-ils vraiment changé ? - Sandrine Nicourd

Transformations et inerties du bénévolat associatif sur la période 1982-2002 - Denis Bernardeau-Moreau et Mathieu Hély

Parcours d'engagement en sociologie - entretien avec Tripier et Claude Dubar

- Le worker center et ses spectres : les conditions d'une mobilisation collective des travailleurs précaires à Chicago - Sébastien Chauvin

Les Motivé-e-s, ou l'innovation prisonnière des règles du jeu politique - Baptiste Giraud

Qu'est-ce qui fait courir les militants de la Ligue communiste révolutionnaire ? - Stéphanie Rizet

La permanence d'un engagement communautaire. Le cas des Scouts et Guides de France - Blaise Barbance et Alexandra Ughetto-Schloupt

Les militants face aux contraintes managériales : le cas de groupes locaux de Handicap International, Sylvain Lefevre et Sylvie Ollitrault

Don de soi ou professionnalisme ? Recrutement et formation à la Délégation Catholique pour la Coopération - Clémence Bosselut

- Du bouche-à-oreille à l'entretien d'embauche : le recrutement dans les associations de commerce équitable - Matthieu Gateau

- Sociologie en Algérie : état des lieux - Mohamed Benguerna et Azzedine Lamria

Les sciences sociales en Algérie. Regards sur les usages de la sociologie - Mohamed Madoui
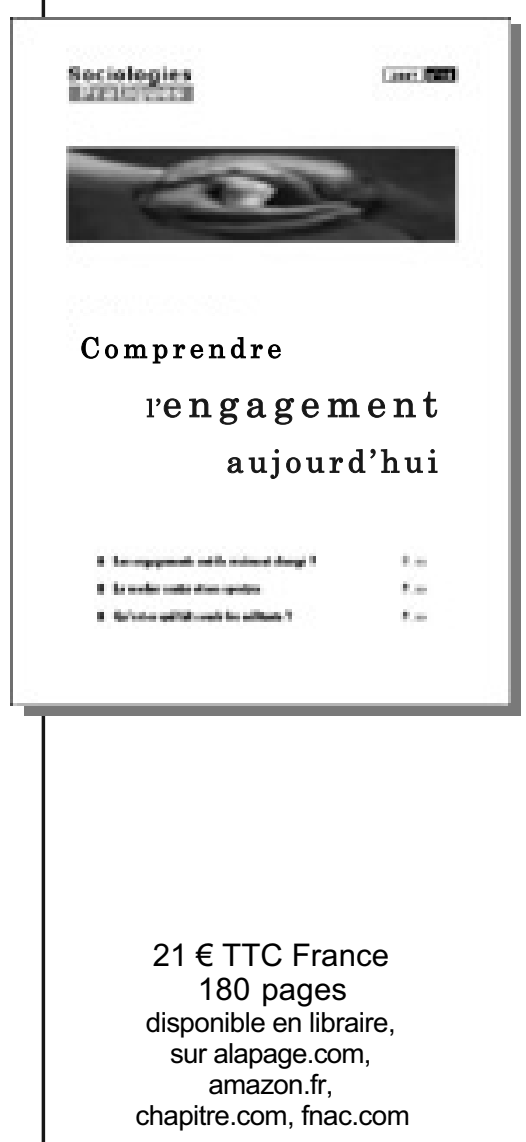

Bon d'abonnement téléchargeable sur www.puf.com

Edité avec le soutien de l'Association des Professionnels en Sociologie de l'Entreprise, du Laboratoire Interdisciplinaire pour la Sociologie Economique et de I'Institut d'Etudes Politiques de Paris

Anciens numéros (achat sur www.sociologies-pratiques.com)

$n^{\circ} 9$ - Alternatives associatives

$n^{\circ} 8$ - Une sociologie en action : l'engagement de Renaud Sainsaulieu

$n^{\circ} 7$ - Gérer l'environnement : le temps de l'action concertée ?

$n^{\circ} 6$ - Les consultants font-ils le monde?

$n^{\circ} 5$ - Mondialisation : les cultures en question

$n^{\circ} 4$ - Diriger des organisations en transformation

$n^{\circ} 3$ - Action dirigeante et gourvernance d'entreprise

$n^{\circ} 2$ - La modernisation du service public

Commande : Tel : 0158103163 -Presses Universitaires de France - Département des revues
6 avenue Reille 75685 Paris Cedex 14 - Réglement par chèque bancaire ou postal à l'ordre de PUF

\begin{tabular}{ccc} 
& Abonnement 2007 (2 numéros) - TVA 5,5\% \\
& Particulier & Institution \\
France & $42,00 €$ TTC & $46,00 €$ TTC \\
Etranger & $49,59 €$ TTC & $54,86 €$ TTC \\
\hline
\end{tabular}

